\title{
Assessing Grid-Accommodable Renewable Capacity with Small-signal Stability Constraints
}

This paper was downloaded from TechRxiv (https://www.techrxiv.org).

\section{LICENSE}

CC BY-NC-SA 4.0

SUBMISSION DATE / POSTED DATE

$17-01-2022$ / 20-01-2022

\section{CITATION}

Yuan, Hui; Xin, Huanhai; Wu, Di; Li, Zhiyi; Zhou, Yuhan; Huang, Linbin (2022): Assessing GridAccommodable Renewable Capacity with Small-signal Stability Constraints. TechRxiv. Preprint. https://doi.org/10.36227/techrxiv.18516275.v1

$\mathrm{DOI}$

10.36227/techrxiv.18516275.v1 


\title{
Assessing Grid-Accommodable Renewable Capacity with Small-signal Stability Constraints
}

\author{
Hui Yuan, Huanhai Xin, Di Wu, Zhiyi Li, Yuhan Zhou, Linbin Huang
}

\begin{abstract}
The increasing penetration of renewable energy resources via power-electronic converters is turning the modern power grid into a multiple-converter system (MCS). In a MCS, the dynamics of converter-based resources (CBRs) are different from those of traditional synchronous machines, which poses great challenges to the grid planning and operation. One of the major challenges is the emerging small-signal stability problems resulting from the interaction between CBRs and the power network. These small-signal stability problems endanger the reliable and stable grid operation. To maintain grid stability, the capacity of CBR generation in the grid may be limited, which impedes the progress towards a sustainable future. To enhance the grid-accommodable capacity (GAC) of renewable generation while maintaining grid stability, this paper presents a semi-definite programming (SDP)-based method to assess GAC with small-signal stability constraints (GAC-SSSC) in a MCS. In the proposed method, the small-signal stability constraints are formulated by the smallest eigenvalue of a pertinent weighted Laplacian matrix of power network, so that the assessment complexity of GAC-SSSC is significantly reduced for a MCS with large-scale CBRs. It is theoretically proved that the derived SDP can find the optimal solution. The efficacy of the proposed method is demonstrated on a 39-bus test system.
\end{abstract}

Index Terms - grid-accommodable capacity, small-signal stability, converter-based resources, semi-definite programming.

\section{INTRODUCTION}

The increasing integration of renewable resources such as wind and solar via power electronic inverters is changing power grid dynamics and challenging the reliable and stable grid operation $^{[1]-[5]}$. To maintain grid reliability and stability, the large-scale generation of inverter-based resources may be curtailed. For example, over $169 \mathrm{TW} \cdot \mathrm{h}$ of wind electricity and $46 \mathrm{TW} \cdot \mathrm{h}$ of photovoltaic electricity in China were curtailed in 2019 to maintain the grid stability. Such a high curtailment level of renewable generation leads to a low utilization efficiency of CBRs. To improve the use rate of integrated CBRs, it is necessary to increase the grid capacity of accommodating renewable generation while maintaining the grid stability. A significant step for this purpose is to determine the grid-accommodable capacity (GAC).

This work is jointly supported by the National Key R\&D Programme of China (No.2017YFB0902000) and the 2018 Science and Technology Programme of Shaanxi Electric Power Corporation.

H. Yuan, H. Xin, Z. Li and Y. Zhou are with the college of electrical engineering, Zhejiang university, Hangzhou 310027, China (Email: xinhh@zju.edu.cn; Yuan_Hui@zju.edu.cn);

D. Wu is with the department of electrical and computer engineering, North Dakota State University, Fargo, 58102, USA.

L. Huang is with the department of information technology and electrical engineering, ETH Zürich, 8092 Zürich, Switzerland
The GAC assessment of CBRs in a multiple-converter system (MCS) have aroused great concerns among academia and industry. The existing assessment methods can be mainly classified into two categories: flexibility planning ${ }^{[6]-[10]}$ and the GAC assessment with static security constraints ${ }^{[1]-[13]}$. The flexibility planning aimed at coordinating renewable resources throughout the system to ensure the power and energy balance with regards to the active-power-output fluctuation of renewable resources ${ }^{[6]-[10]}$. The GAC assessment with static security constraint is to prevent voltage violation ${ }^{[11]-[12]}$, and steady-state instability ${ }^{[13]}$, wherein the analysis is based on the system's power flow equations.

Most of the previous works did not consider the small-signal stability constraint, which is in fact one of the key factors that limit the GAC of CBRs in modern power systems ${ }^{[14]-[19]}$. For instance, Refs. [14]-[16] demonstrated that the increasing capacities of grid-following inverters with a phase-locked loop (PLL) in a single-converter system (SCS) will deteriorate the small-signal stability. Furthermore, the similar conclusion was found in $\mathrm{MCSs}^{[17]-[19]}$. We note that grid-forming inverters may not need a PLL for grid synchronization ${ }^{[20]}$. Thus, interfacing CBRs with the grid via grid-forming inverters may enhance GAC while maintaining the small-signal stability in a MCS. However, the technologies for grid-forming inverters are still under developing for practical applications ${ }^{[20]}$. Currently, the majority of CBRs are interfaced with the grid using grid-following inverters. In this context, the small-signal stability problem will still be one of key factors limiting the GAC of CBRs in MCSs.

To assess GAC with the small-signal stability constraints (GAC-SSSC) in a MCS dominated by grid-following inverters, this paper presents a semi-definite programming (SDP)-based method. To reduce the complexity of GAC-SSSC assessment, the small-signal stability constraints in the proposed method are formulated based on the smallest eigenvalue of a weighted Laplacian matrix of the power network. This method avoids using the damping ratio, which is a traditional way to quantify the small-signal stability in a power system that is dominated by synchronous generators ${ }^{[21]}$. By comparison, the proposed SDP-based method is suitable for assessing the GAC-SSSC in a MCS with large-scale CBRs. Moreover, we prove that there exists the optimal solution to the derived SDP.

The rest of this paper is organized as follows. In Section II the GAC-SSSC problem is described. In Section III, the small-signal stability of MCSs is analyzed. In Section IV, the proposed SDP-based method is presented to assess GAC-SSSC in MCSs. In Section V, the SDP-based method is validated. 
Section VI draws the conclusions.

\section{II.PROBLEM FORMULATION}

Consider a power network with $n+m+1$ nodes as shown in Fig. 1, wherein nodes $1 \sim n$ are connected to CBRs, node $n+m+1$ is connected to the external grid (simplified as an ideal source), and the remaining nodes are passive nodes ${ }^{[22]}$. When the control parameters of CBRs and the power network are given, the GAC-SSSC problem can be generally described by

$$
\begin{aligned}
& \max \sum_{i=1}^{n} S_{B i} \\
& \text { s.t. }\left\{\begin{array}{l}
S_{B i} \geq 0, \quad i=1, \ldots, n \\
\text { Small-signal stability constraints }
\end{array}\right.
\end{aligned}
$$

where $S_{B i}$ is the capacity of CBRi. In (1), the GAC of CBRs is the maximum allowable sum of all CBRs' capacities.

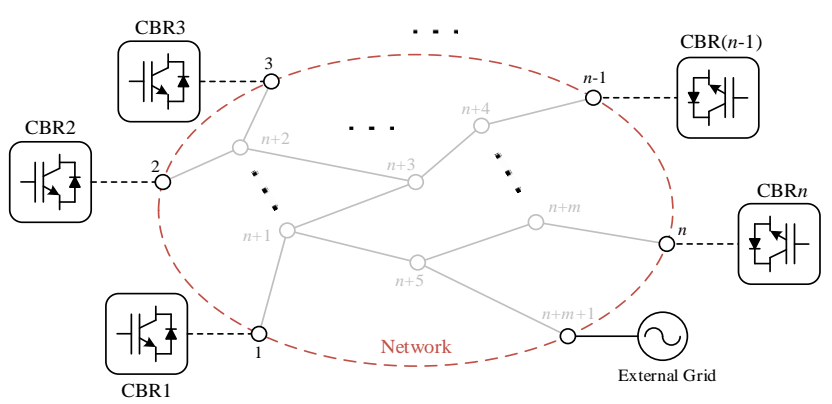

Fig. 1. A network of CBRs integrated at nodes $1 \sim n$.

For the GAC-SSSC in (1), the quantification of small-signal stability is important for formulating the small-signal stability constraints. Traditionally, the small-signal stability is commonly evaluated by the damping ratio. However, it is in general difficult and not tractable to use the damping ratio to formulate the small-signal stability constraints (SSSC) in (1) for the GAC-SSSC assessment of MCSs with large-scale CBRs due to 1) the high dimension of the system's state matrix required to derive damping ratio ${ }^{[23]}$ and 2) the strong nonlinearity between the damping ratio and the CBRs' capacity variables in (1).

To address these challenges, we define the damping ratio of the system's dominant eigenvalues as $\zeta_{\omega}=-\sigma / \sqrt{\sigma^{2}+\omega^{2}}$, where $\sigma$ and $\omega$ are the real and imaginary parts of the dominant eigenvalues $s_{\omega}=\sigma \pm j \omega$. To calculate damping ratio $\zeta_{\omega}$, we need to obtain the system's state matrix $A_{w}$, which is associated with the system's state variables including the state variables of CBRs and the power network. Thus, when large-scale CBRs with complex control configurations are integrated in the power network, the dimension of state matrix $A_{w}$ is very high, which thus complicates the damping ratio calculation. For example, for the power network shown in Fig. 1, when two types of widely-used active power controls are used, as shown in Fig. 2 (i.e., dc-voltage control or constant active power control ${ }^{[15]}$ ), each CBR with constant active power control (or dc-voltage control) has 11 (or 12) state variables. As a result, the dimension of the state matrix $A_{w}$ is larger than $11 n$ when considering the network dynamics. Therefore, the complexity of the damping ratio calculation increases with the increase of the number of CBRs in the MCS. In addition, there is a nonlinear relationship between the eigenvalues of state matrix $A_{w}$ and CBR capacities $S_{B i},(i=1, \ldots, n)$, which thus leads to a nonlinear relationship between the damping ratio and CBR capacities $S_{B i}$ as well. When the SSSC in (1) is formulated based on the damping ratio, the GAC-SSSC optimization problem in (1) is nonlinear, and its convexity is unknown. Therefore, it is hard to find the global optimal solution to (1). To reduce the complexity of solving optimization problem (1), we will formulate its SSSC in an alternative way based on the small-signal stability analysis of MCS in next section.

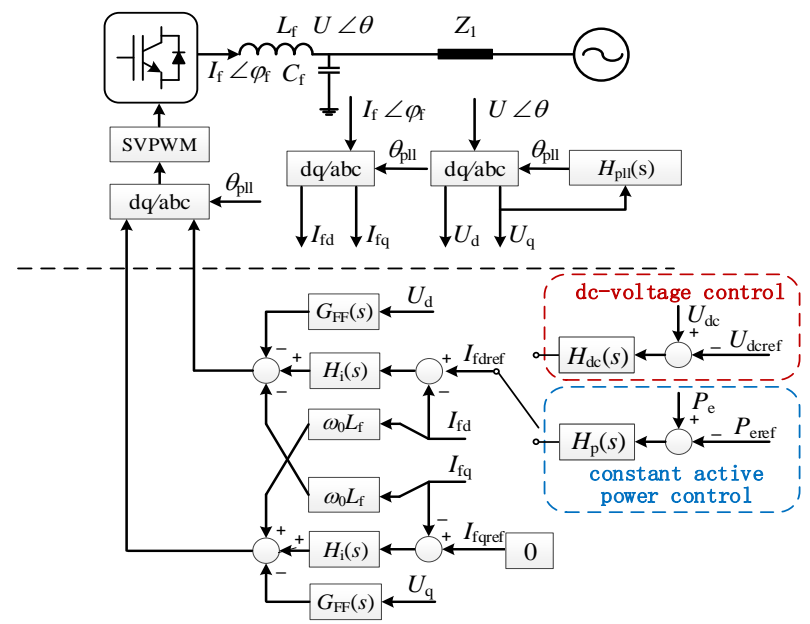

Fig. 2 Block diagram of CBR with PLL-based vector control in a SCS.

\section{SMALL-SIGNAL STABILITY OF MCSS}

\section{A. Impedance-based Modelling for MCSs}

Consider a structure-preserving MCS as shown in Fig. 1, where the control diagram of CBRs in the system is shown in Fig. 2, and the control parameters are given in Appendix. Since the impedance-based modelling is commonly used for the small-signal stability analysis of MCSs, the MCS can be represented by a frequency-domain (s-domain) impedance-based model, which includes the models at the CBR side and ac network side

$$
\begin{aligned}
\Delta \mathbf{I}_{x y} & =-\mathbf{Y}_{\mathrm{CBR} m}(s) \Delta \mathbf{U}_{x y} \\
\Delta \mathbf{I}_{x y} & =\mathbf{Y}_{\text {netm }}(s) \Delta \mathbf{U}_{x y}
\end{aligned}
$$

where $\Delta$ denotes the perturbed value of a variable; $\mathbf{I}_{x y}$ and $\mathbf{U}_{x y}$ are the vectors representing the current injections and terminal voltages at the CBR nodes in the global $x y$-frame, respectively; $\mathbf{Y}_{\mathrm{CBR} m}(s)$ and $\mathbf{Y}_{\text {net } m}(s)$ represent the frequency-domain admittance matrices of the CBRs and the network, respectively.

Based on (2) and (3), the closed-loop characteristic equation of the MCS is derived as

$$
\operatorname{det}\left(\mathbf{Y}_{\mathrm{CBR} m}(s)+\mathbf{Y}_{\text {net } m}(s)\right)=0
$$

where $\operatorname{det}(\cdot) \operatorname{denotes}$ the determinant of a matrix.

To analyze the small-signal stability of the MCS based on (4), we derive the detailed expression of admittance matrices $\mathbf{Y}_{\mathrm{CBR} m}(s)$ and $\mathbf{Y}_{\mathrm{net} m}(s)$ in (4). For the network-side impedance modeling, when the network is represented by the lines that have the same ratio $\tau$ of line resistance $R$ to line inductance $L$ 
(i.e., $\tau=R / L$ ), the admittance matrix $\mathbf{Y}_{\text {net } m}(s)$ in (4) can be represented by ${ }^{[22]}$

$$
\begin{gathered}
\mathbf{Y}_{\text {net } m}(s)=\mathbf{B}_{r e d} \otimes \gamma(s), \gamma(s)=\left[\begin{array}{cc}
\beta(s) & \alpha(s) \\
-\alpha(s) & \beta(s)
\end{array}\right] \\
\mathbf{B}_{\text {red }}=\left[B_{i j}\right]_{n \times n}, B_{i j}= \begin{cases}\sum_{j=1}^{n} 1 /\left(\omega_{0} L_{i j}\right), & j=i \\
-1 /\left(\omega_{0} L_{i j}\right), & j \neq i\end{cases}
\end{gathered}
$$

where $\otimes$ is the Kronecker product ${ }^{[24]} ; \mathbf{B}_{\text {red }} \in \mathbb{R}^{n \times n}$ is the node-reduced susceptance matrix, in which the passive nodes are eliminated; $B_{i j}$ and $L_{i j}$ are the susceptance and inductance of the line between node $i$ and node $j$, respectively; $\omega_{0}$ is rated synchronous frequency; $\alpha(s)=\omega_{0}^{2} /\left[\left(s+\tau \omega_{0}\right)^{2}+\omega_{0}^{2}\right]$ and $\beta(s)=\omega_{0}\left(s+\tau \omega_{0}\right) /\left[\left(s+\tau \omega_{0}\right)^{2}+\omega_{0}^{2}\right]$.

For CBRs with different control strategies and parameters, admittance matrix $\mathbf{Y}_{\mathrm{CBR} m}(s)$ in (4) can be expressed by

$$
\mathbf{Y}_{\mathrm{CBR} m}(s)=\operatorname{diag}\left(S_{B i} \mathbf{G}_{i x y}(s)\right)
$$

where $\operatorname{diag}(\cdot)$ denotes a diagonal matrix; $S_{\mathrm{B} i} \mathbf{G}_{i x y}(s)$ is the $i$ th diagonal submatrix; $\mathbf{G}_{i x y}(s)$ is the admittance matrix of the $i$ th CBR normalized at its rated capacity in the global $x y$-frame. Specifically, the impedance model of the $i$ th CBR in the global $x y$-frame can be expressed as ${ }^{[22]}$

$$
\left[\begin{array}{c}
\Delta I_{i x} \\
\Delta I_{i y}
\end{array}\right]=-S_{B i} \underbrace{\left[\begin{array}{ll}
G_{i x x}(s) & G_{i x y}(s) \\
G_{i y x}(s) & G_{i y y}(s)
\end{array}\right]}_{\mathbf{G}_{i y y}(s)}\left[\begin{array}{c}
\Delta U_{i x} \\
\Delta U_{i y}
\end{array}\right]
$$

where the detailed expression of $G_{i x x}(s), G_{i x y}(s), G_{i y x}(s)$ and $G_{i y y}(s)$ can be founded in [25]; $I_{i x}$ and $I_{i y}$ are the $x$-axis and $y$-axis elements of the current injection at the $i$ th CBR node; $U_{i x}$ and $U_{i y}$ are the $x$-axis and $y$-axis elements of the terminal voltage at the $i$ th CBR node.

By submitting (5) and (7) into (4), the characteristic equation of the MCS can be further represented by:

$$
\begin{gathered}
\operatorname{det}\left\{\left(\mathbf{S}_{B} \otimes \mathbf{I}_{2}\right) \mathbf{C}(s)\left(\mathbf{I}_{n} \otimes \gamma(s)\right)\right\}=0 \\
\mathbf{C}(s)=\operatorname{diag}\left(\mathbf{Y}_{i}(s)\right)+\left(\mathbf{S}_{B}^{-1} \mathbf{B}_{r e d}\right) \otimes \mathbf{I}_{2} \\
\mathbf{Y}_{i}(s)=\mathbf{G}_{i x y}(s) \boldsymbol{\gamma}^{-1}(s)
\end{gathered}
$$

where $\mathbf{S}_{B}=\operatorname{diag}\left(S_{B i}\right)$ is a diagonal matrix, in which the diagonal element is $S_{B i} ; \mathbf{S}_{B}^{-1} \mathbf{B}_{\text {red }}$ is a weighted and Kron-reduced Laplacian matrix ${ }^{[22]}$; and $\mathbf{I}_{n} \in \mathbb{R}^{n \times n}$ denotes the identity matrix.

Due to $\operatorname{det}\left(\boldsymbol{S}_{B}\right) \neq 0$ and $\operatorname{det}(\gamma(s))=\alpha^{2}(s)+\beta^{2}(s) \neq 0$, the characteristic equation (9) is equivalently rewritten by

$$
\operatorname{det}(\mathbf{C}(s))=\operatorname{det}\left[\operatorname{diag}\left(\mathbf{Y}_{i}(s)\right)+\left(\mathbf{S}_{B}^{-1} \mathbf{B}_{r e d}\right) \otimes \mathbf{I}_{2}\right]=0
$$

where $\mathbf{C}(s)$ denotes the closed-loop transfer function matrix.

\section{B. Small-Signal Stability Analysis of MCSs}

Based on (12), the small-signal stability of the MCS can be analyzed. To this end, we consider two scenarios: 1) the MCS is a homogeneous system, where all CBRs have the same control strategies and control parameters at its own rated capacity in the MCS, and 2) the MCS is a heterogeneous system, where CBRs have different control parameters and control strategies.
In the homogeneous system, $\mathbf{Y}_{i}(s)$ in (12) for all CBRs are identical. Under this condition, (12) can thus be rewritten as

$$
\begin{gathered}
\operatorname{det}(\mathbf{C}(s))=\operatorname{det}\left[\mathbf{I}_{n} \otimes \mathbf{Y}(s)+\left(\mathbf{S}_{B}^{-1} \mathbf{B}_{r e d}\right) \otimes \mathbf{I}_{2}\right]=0 \\
\mathbf{Y}(s)=\mathbf{G}_{x y}(s) \boldsymbol{\gamma}^{-1}(s)
\end{gathered}
$$

where $\mathbf{G}_{x y}(s)$ is the impedance matrix of CBRs at its rated capacity in the homogeneous MCS.

The characteristic equation (13) can be further represented by (15) when left-multiplying (13) by $\mathbf{I}_{2} \otimes \mathbf{P}$, and right-multiplying (13) by $\mathbf{I}_{2} \otimes \mathbf{P}^{-1}$ (matrix $\mathbf{P}$ decomposes $\mathbf{S}_{B}^{-1} \mathbf{B}_{\text {red }} \quad$ as $\quad \mathbf{P}\left(\mathbf{S}_{B}^{-1} \mathbf{B}_{\text {red }}\right) \mathbf{P}^{-1}=\operatorname{diag}\left(\lambda_{i}\right)$, wherein $0<\lambda_{1}<\lambda_{2} \leq \cdots$ $\left.\leq \lambda_{n}{ }^{[22]}\right)$

$$
\begin{gathered}
\operatorname{det}(\mathbf{C}(s))=\prod_{i=1}^{n} \operatorname{det}\left(\mathbf{C}_{i}(s)\right)=0 \\
\mathbf{C}_{i}(s)=\mathbf{Y}(s)+\lambda_{i} \mathbf{I}_{2}
\end{gathered}
$$

Equation (15) shows that a homogeneous MCS can be decoupled into $n$ dynamically independent SCSs for the small-signal stability analysis. For each SCS, its characteristic equation can be represented by $\operatorname{det}\left(\mathbf{C}_{i}(s)\right)=0$, which indicates the stability of each SCS depends on eigenvalues $\lambda_{i}$ of the weighted Laplacian matrix $\mathbf{S}_{B}^{-1} \mathbf{B}_{\text {red }}$ when the control strategies and control parameters of CBRs at their own rated capacities in the MCS are given. Thus, the small-signal stability of the entire MCS depends on the critical SCS that has the smallest eigenvalue $\lambda_{1}$ of the weighted Laplacian matrix $\mathbf{S}_{B}^{-1} \mathbf{B}_{\text {red }}$ of the power network, i.e., smallest network eigenvalue. That is, a larger $\lambda_{1}$ indicates that the MCS becomes more stable; a lower $\lambda_{1}$ increases the risk of instability. Therefore, the smallest network eigenvalue $\lambda_{1}$ can be used to quantify the small-signal stability instead of damping ratio $\zeta_{\omega}$ in the MCSs. In [22], $\lambda_{1}$ is defined as generalized short circuit ratio to quantify grid strength for the small-signal stability analysis of the MCS.

Different from homogeneous MCSs, a heterogeneous MCS is composed of CBRs that have different control parameters and control strategies. That is, the admittance matrix $\mathbf{Y}_{i}(s)$ for CBRs in (12) are different. Under this condition, the characteristic equation (12) cannot be directly decomposed, but the smallest network eigenvalue $\lambda_{1}$ can still be used for the small-signal stability analysis of heterogeneous MCSs. This can be proved based on the matrix perturbation theory. More specifically, an equivalent homogeneous MCS for a heterogeneous MCS can be obtained, and the closed-loop transfer function matrix $\overline{\mathbf{C}}(s)$ of the equivalent homogeneous MCS is represented by

$$
\begin{gathered}
\overline{\mathbf{C}}(s)=\mathbf{I}_{n} \otimes \overline{\mathbf{Y}}(s)+\left(\mathbf{S}_{B}^{-1} \mathbf{B}_{r e d}\right) \otimes \mathbf{I}_{2} \\
\overline{\mathbf{Y}}(s)=\overline{\mathbf{G}}_{x y}(s) \boldsymbol{\gamma}^{-1}(s) \\
\overline{\mathbf{G}}_{x y}(s)=\sum_{i=1}^{n} p_{i} \mathbf{G}_{i x y}(s) \\
p_{i}=u_{i 1} v_{i 1}
\end{gathered}
$$

where $u_{i 1}$ and $v_{i 1}$ are the $i$ th element of $\boldsymbol{u}_{1}^{T}$ and $\boldsymbol{v}_{1} ; \boldsymbol{u}_{1}^{T}$ and $\boldsymbol{v}_{1}$ are the normalized left and right eigenvectors of the weighted Laplacian matrix $\mathbf{S}_{B}^{-1} \mathbf{B}_{\text {red }}$ for the smallest network eigenvalue $\lambda_{1}$, respectively; $\overline{\mathbf{G}}_{x y}(s)$ is the CBR impedance matrix of the equivalent homogeneous MCS, which is the weighted sum of $n$ 
CBR dynamics in a heterogeneous MCS; $p_{i},(i=1, \ldots, n)$ is the participation factor, and satisfies $\sum_{i=1}^{n} p_{i}=1, \quad 0<p_{i}<1$.

The relation in the dominant eigenvalues between the heterogeneous MCS and its equivalent homogeneous MCS can be described as Lemma 1:

Lemma 1: Let $c(s)$ and $\bar{c}(s)$ be the dominant eigenvalue functions of the heterogeneous MCS and its equivalent homogeneous MCS, respectively, and they are pertinent to the system dominant eigenvalues. Then, the loci of $c(s)$ and $\bar{c}(s)$ have the following relationship:

$$
c(s)=\bar{c}(s)+\boldsymbol{o}(\|\mathbf{C}(s)-\overline{\mathbf{C}}(s)\|)
$$

where $\boldsymbol{o}(\|\mathbf{C}(s)-\overline{\mathbf{C}}(s)\|)$ denotes the second-order and higherorder errors.

Proof. As discussed in Section III.B, the stability of an equivalent homogeneous MCS in (17) can be represented by its decoupled critical SCS that has the smallest network eigenvalue $\lambda_{1}$. The characteristic equation of this SCS can be written as:

$$
\operatorname{det}\left(\overline{\mathrm{C}}_{1}(s)\right)=\operatorname{det}\left(\overline{\mathbf{Y}}(s)+\lambda_{1} \mathbf{I}_{2}\right)=0
$$

Then, we can conclude that $\bar{c}(s)$ is also the dominant eigenvalue function of the critical SCS with smallest network eigenvalue $\lambda_{1}$. Let $\boldsymbol{\mu}^{T}(s)$ and $\boldsymbol{v}(s)$ be the normalized left and right eigenvectors of $\overline{\mathbf{Y}}(s)$ for eigenvalue function $\rho(s)$, which is pertinent to the system dominant eigenvalues, i.e.,

$$
\rho(s)=\boldsymbol{\mu}^{T}(s) \overline{\mathbf{Y}}(s) \boldsymbol{v}(s)
$$

Combining (22) and (23), the dominant eigenvalue function $\bar{c}(s)$ for $\overline{\mathrm{C}}_{1}(s)$ is expressed as

$$
\bar{c}(s)=\boldsymbol{\mu}^{T}(s) \overline{\mathbf{C}}_{1}(s) \boldsymbol{v}(s)=\rho(s)+\lambda_{1}
$$

Based on (17) and (24), the normalized left and right eigenvectors of the transfer function matrix $\overline{\mathbf{C}}(s)$ for $\bar{c}(s)$ can be expressed as $\boldsymbol{u}_{1}^{T} \otimes \boldsymbol{\mu}^{T}(s)$ and $\boldsymbol{v}_{1} \otimes \boldsymbol{v}(s)$. That is,

$$
\begin{gathered}
\bar{c}(s)=\left[\boldsymbol{u}_{1}^{T} \otimes \boldsymbol{\mu}^{T}(s)\right] \overline{\mathbf{C}}(s)\left[\boldsymbol{v}_{1} \otimes \boldsymbol{v}(s)\right] \\
1=\left[\boldsymbol{u}_{1}^{T} \otimes \boldsymbol{\mu}^{T}(s)\right]\left[\boldsymbol{v}_{1} \otimes \boldsymbol{v}(s)\right]
\end{gathered}
$$

Here, $\mathbf{C}(s)$ can be viewed as a perturbation of $\overline{\mathbf{C}}(s)$. Based on the matrix perturbation theory (Theorem $2.3^{[26]}$ ), the loci of the dominant eigenvalue function $c(s)$ for $\mathbf{C}(s)$ is given as

$$
\begin{aligned}
& c(s)=\left[\boldsymbol{u}_{1}^{T} \otimes \boldsymbol{\mu}^{T}(s)\right] \mathbf{C}(s)\left[\boldsymbol{v}_{1} \otimes \boldsymbol{v}(s)\right]+\boldsymbol{o}(\|\mathbf{C}(s)-\overline{\mathbf{C}}(s)\|) \\
& =\left[\boldsymbol{u}_{1}^{T} \otimes \boldsymbol{\mu}^{T}(s)\right] \operatorname{diag}\left(\mathbf{Y}_{i}(s)\right)\left[\boldsymbol{v}_{1} \otimes \boldsymbol{v}(s)\right]+ \\
& {\left[\boldsymbol{u}_{1}^{T}\left(\mathbf{S}_{B}^{-1} \mathbf{B}_{r e d}\right) \boldsymbol{v}_{1}\right] \otimes\left[\boldsymbol{\mu}^{T}(s) \mathbf{I}_{2} \boldsymbol{v}(s)\right]+\boldsymbol{o}(\|\mathbf{C}(s)-\overline{\mathbf{C}}(s)\|)} \\
& =\rho(s)+\lambda_{1}+\boldsymbol{o}(\|\mathbf{C}(s)-\overline{\mathbf{C}}(s)\|) \\
& =\bar{c}(s)+\boldsymbol{o}(\|\mathbf{C}(s)-\overline{\mathbf{C}}(s)\|)
\end{aligned}
$$

This concludes the proof $\mathbf{a}$.

Lemma 1 shows that the dominant eigenvalues of the heterogeneous MCS can be approximated by its equivalent homogeneous MCS (17), if $\boldsymbol{o}(\|\mathbf{C}(s)-\overline{\mathbf{C}}(s)\|)$ is small enough.

In other words, the small-signal stability of a heterogeneous MCS can be approximated by that of its equivalent homogeneous MCS. Since the stability of the equivalent homogeneous MCS can be characterized by the smallest network eigenvalue $\lambda_{1}$ as shown in (22), the smallest network eigenvalue $\lambda_{1}$ can also be used to evaluate the small-signal stability of the heterogeneous MCS.

The small-signal stability analysis of MCSs also shows that the small-signal stability of the heterogeneous MCS is bounded by the dynamics of all CBRs. According to lemma 1, the small-signal stability of the heterogeneous MCS can be characterized by its equivalent homogeneous MCS. The small-signal stability of the equivalent homogeneous MCS depends on its critical SCS (i.e., $\mathrm{SCS}_{1}$ ), which is shown in (22). As illustrated in Fig. 3 (a), the critical SCS is connected to the grid through the equivalent line admittance $\lambda_{1}$ (i.e., the smallest network eigenvalue) and its dynamics depend on the weighted sum of $n$ CBR dynamics, which is shown in (19).

To evaluate the small signal stability of the critical SCS, we define $n$ equivalent SCSs, as illustrated in Fig. 3(b), where each equivalent SCS has different CBR configurations but the same smallest network eigenvalue $\lambda_{1}$ as the equivalent line admittance connected to the grid. The stability of these $n$ equivalent SCSs is ranked in terms of their damping ratios in the order from the smallest to the largest, i.e., $\mathrm{SCS}_{1,1}, \mathrm{SCS}_{1,2}, \ldots$, $\mathrm{SCS}_{1, n}$. As shown in Fig. 3 (a), we can see that in the critical SCS if the participation factor $p_{i}$ of the CBR's dynamic (same as that of $\mathrm{SCS}_{1,1}$ ) is larger, the stability of the critical SCS will tend to be unstable; otherwise, if the participation factor $p_{i}$ of the CBR's dynamic (same as that of $\mathrm{SCS}_{1, \mathrm{n}}$ ) is larger, the stability of the critical SCS will become better. Since the participation factor $p_{i}$ of the CBRi's dynamic $\mathbf{G}_{i}(s)$ in the critical SCS satisfies $\sum_{i=1}^{n} p_{i}=1,0<p_{i}<1$, the extreme cases for the critical SCS with given $\lambda_{1}$ and CBRs' dynamics are that: 1) when the participation factor $p_{i}$ of the CBR's dynamic (same as that of $\mathrm{SCS}_{1,1}$ ) is one and the other $p_{i}$ are all zero (i.e., $\mathrm{SCS}_{1,1}$ ), the stability of the system is worst; 2) when the participation factor $p_{i}$ of the CBR's dynamic (same as that of $\mathrm{SCS}_{1, \mathrm{n}}$ ) is one and the other $p_{i}$ are all zero (i.e., $\mathrm{SCS}_{1, \mathrm{n}}$ ), the stability of the system is best. That is, the stability of the critical SCS (or the equivalent homogeneous MCS) is bounded by equivalent $\mathrm{SCS}_{1,1}$ and $\mathrm{SCS}_{1, \mathrm{n}}$. Since the small-signal stability of a heterogeneous MCS can be characterized by its equivalent homogeneous MCS, the small-signal stability of a heterogeneous MCS is bounded by the equivalent $\mathrm{SCS}_{1,1}$ and $\mathrm{SCS}_{1, \mathrm{n}}$, which will be further demonstrated in Section V.

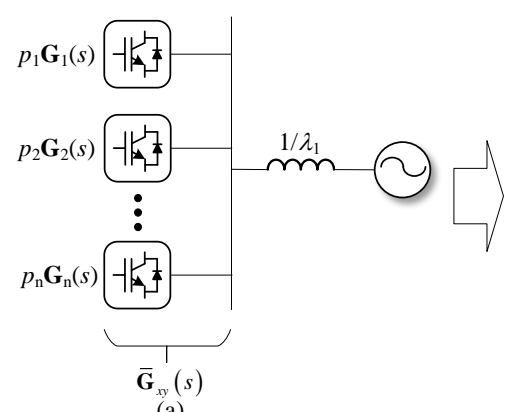

$\mathbf{G}_{x y}(s)$
(a)

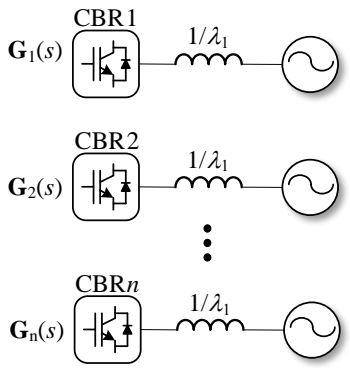

(b)
Fig. 3 Illustration of the critical SCS (or $\mathrm{SCS}_{1}$ ) and its equivalent SCSs: (a) the critical SCS decoupled from the equivalent homogeneous MCS; (b) $n$ equivalent SCSs of the critical SCS. 


\section{PRoposed SDP-BASEd Method FOR GAC-SSSC ASSESSMENT IN MCSS}

Based on the theoretical results in the previous section, in this section, the optimization problem (1) will be first reformulated for GAC-SSSC assessment and then be converted into an SDP problem for an efficient solution. Also, we will prove the feasibility of finding the optimal solution of the established SDP.

\section{A. GAC-SSSC Assessment}

As discussed in Section III, the small-signal stability of MCSs can be evaluated by the smallest network eigenvalue $\lambda_{1}$. When the small-signal stability constraint in (1) is formulated based on the smallest network eigenvalue $\lambda_{1}$, the optimization problem (1) can be reformulated as

$$
\begin{gathered}
\max \sum_{i=1}^{n} S_{B i} \\
\text { s.t. }\left\{\begin{array}{l}
S_{B i} \geq 0, \quad i=1, \ldots, n \\
\lambda_{1} \geq \lambda_{1 \text { min }}
\end{array}\right.
\end{gathered}
$$

where $\lambda_{1 \min }$ is the required small-signal stability limit.

To solve optimization problem (28), it needs to determine the value of $\lambda_{1 \mathrm{~min}}$. Since the small-signal stability of the MCS considers two scenarios of homogeneous and heterogeneous MCSs, we further discuss how to determine the $\lambda_{1 \min }$ in (28) in the two scenarios. In homogeneous MCSs, the $\lambda_{1 \min }$ in (28) can be determined by the characteristic equation of (16). That is, the $\lambda_{1 \text { min }}$ is the solution to $\operatorname{det}\left(\mathbf{Y}(s)+\lambda_{1 \min } \mathbf{I}_{2}\right)=0$, when the system damping ratio reaches the required threshold $\zeta_{\text {min }}$.

In a heterogeneous MCS, the $\lambda_{1 \min }$ can be evaluated by the characteristic equation $\operatorname{det}\left(\overline{\mathbf{Y}}(s)+\lambda_{1 \min } \mathbf{I}_{2}\right)=0$ referring to (17) with a given damping ratio threshold $\zeta_{\min }$. However, in heterogeneous MCSs, evaluating the stability limit $\lambda_{1 \min }$ in (28) needs to construct the detailed models for the equivalent SCSs based on (19). To simplify this evaluation, we can evaluate the upper limit of the $\lambda_{1 \min }$. As discussed in Section III.B, the small-signal stability of a heterogeneous MCS is bounded by the dynamics of all CBRs. That is, the $\lambda_{1 \min }$ in the heterogeneous MCS is in the range of $\lambda^{\mathrm{L}}{ }_{1 \mathrm{~min}} \leq \lambda_{1 \mathrm{~min}} \leq \lambda^{\mathrm{U}}{ }_{1 \mathrm{~min}}$, wherein $\lambda^{\mathrm{L}}{ }_{1 \mathrm{~min}}$ (or $\lambda^{\mathrm{U}}{ }_{1 \mathrm{~min}}$ ) is the lower (or upper) limit of $\lambda_{1 \mathrm{~min}}$, which is the corresponding value of $\lambda_{1}$ in the most stable equivalent SCS, i.e., $\mathrm{SCS}_{1, \mathrm{n}}$, (or the most unstable equivalent SCS, i.e., $\mathrm{SCS}_{1,1}$ ), when the system damping ratio reaches the given threshold $\zeta_{\min }$. To avoid the construction of the detailed models for the equivalent SCSs based on (19) to evaluate the $\lambda_{1 \text { min }}$ using $\operatorname{det}\left(\overline{\mathbf{Y}}(s)+\lambda_{1 \min } \mathbf{I}_{2}\right)=0$, we can evaluate the $\lambda^{\mathrm{U}}{ }_{1 \mathrm{~min}}$, since $\lambda_{1}>\lambda^{\mathrm{U}}{ }_{1 \text { min }}$ means $\lambda_{1}>\lambda_{1 \text { min. }}$. The optimization problem (28) can thus be rewritten as:

$$
\begin{gathered}
\max \sum_{i=1}^{n} S_{B i} \\
\text { s.t. }\left\{\begin{array}{l}
S_{B i} \geq 0, \quad i=1, \ldots, n \\
\lambda_{1} \geq \lambda_{1 \text { min }}^{\mathrm{U}}
\end{array}\right.
\end{gathered}
$$

In (29), the $\lambda^{\mathrm{U}}{ }_{1 \text { min }}$ can be determined in the following way: for a heterogeneous MCS with $n$ different CBRs, $n$ small-signal stability limits (defined as $\lambda_{1 \min 1}, \ldots, \lambda_{1 \min n}$ ) can be obtained by $n$ SCSs' close-loop characteristic equations $\operatorname{det}\left(\mathbf{Y}_{i}(s)+\lambda_{1 \min i} \mathbf{I}_{2}\right)=0$ when the system stability reaches $\zeta_{\min }$, wherein $\mathbf{Y}_{i}(s)=\mathbf{G}_{i x y}(s) \gamma$ ${ }^{-1}(s)$, and $\mathbf{G}_{i x y}(s)$ is the impedance matrix of an arbitrary CBR in the heterogeneous MCS. Then, $\lambda^{\mathrm{U}}{ }_{1 \min }$ is the largest one among all $\lambda_{1 \min i},(i=1, \ldots, n)$. Note that for a homogeneous MCS, (28) and (29) have the same solutions because $\lambda_{1 \min }$ in (28) and the $\lambda^{\mathrm{U}}{ }_{1 \min }$ in (29) are identical. However, for a heterogeneous MCS, the solution to (29) can be considered as a near-optimal solution to (28), since $\lambda^{\mathrm{U}}{ }_{\text {min }}$ in (29) is the upper limit of $\lambda_{1 \min }$ in (28).

Remark 1. The complex GAC-SSSC assessment in MCSs can be simplified as two sequentially-solved sub-problems to avoid establishing the detailed model for MCSs:

1)Determine the $\lambda_{1 \min }$ in homogeneous MCSs or the $\lambda^{\mathrm{U}}{ }_{1 \min }$ in heterogeneous MCSs;

2) Solve (29) to determine the GAC-SSSC in homogeneous or heterogeneous MCSs.

The optimization problem (29) cannot consider a case, where the capacities of partial CBRs are zero when coordinating the capacities of all CBRs in a MCS to determine the GAC-SSSC, which is demonstrated in Section V. When $S_{\mathrm{B} i}=0, i \in\{1, \ldots, n\}$, the diagonal matrix $\mathbf{S}_{\mathrm{B}}$ is a positive semi-definite matrix rather than a positive definite matrix. Thus, $\mathbf{S}_{\mathrm{B}}$ is irreversible and the $\lambda_{1}$ cannot be calculated by $\mathbf{S}_{B}^{-1} \mathbf{B}_{\text {red }}$ in (29). To address this issue, the optimization problem (29) will be reformulated by the following lemma 2 .

Lemma 2: For a power network with $n$ nodes for potential CBR integration, $\boldsymbol{B}_{\text {red }}\left(\boldsymbol{Z}_{r e d}\right)$ is defined as the node-reduced suceptance (reactance) matrix, in which only the $n$ nodes remain. It is assumed that nodes $1 \sim x,(i \in\{1, \ldots, n\})$ are placed with CBRs that have capacities larger than zeros (i.e., $S_{\mathrm{B} i}$, $i=1, \ldots, x$ and $\left.S_{\mathrm{B} i}>0\right)$, and $\boldsymbol{B}_{\text {redx }}$ is defined as another node-reduced admittance matrix, in which nodes $1 \sim x$ remained. Then, it satisfies that the smallest eigenvalue of matrix $\mathbf{S}_{B x}^{-1} \mathbf{B}_{\text {redx }}$ (i.e., $\lambda_{\min }\left(\mathbf{S}_{B x}^{-1} \mathbf{B}_{\text {redx }}\right)$ ) is the reciprocal of the maximal eigenvalue of matrix $\mathbf{Z}_{r e d} \mathbf{S}_{B}$ (i.e., $\lambda_{\max }^{-1}\left(\mathbf{Z}_{r e d} \mathbf{S}_{B}\right)$. That is,

$$
\begin{gathered}
\lambda_{\min }\left(\mathbf{S}_{B x}^{-1} \mathbf{B}_{\text {redx }}\right)=\lambda_{\max }^{-1}\left(\mathbf{Z}_{\text {red }} \mathbf{S}_{B}\right) \\
\mathbf{Z}_{r e d}^{-1}=\mathbf{B}_{\text {red }}=\left[\begin{array}{ll}
\mathbf{B}_{11} & \mathbf{B}_{12} \\
\mathbf{B}_{21} & \mathbf{B}_{22}
\end{array}\right] \\
\mathbf{S}_{B}=\left[\begin{array}{ll}
\mathbf{S}_{B x} & \\
& \mathbf{0}
\end{array}\right] \\
\mathbf{B}_{\text {redx }}=\mathbf{B}_{11}-\mathbf{B}_{12} \mathbf{B}_{22}^{-1} \mathbf{B}_{21}
\end{gathered}
$$

where $\mathbf{Z}_{\text {red }} \in \mathbb{R}^{n \times n}$ and $\mathbf{B}_{\text {red }} \in \mathbb{R}^{n \times n}$ are the node-reduced reactance matrix and susceptance matrix, respectively; $\mathbf{B}_{11} \in \mathbb{R}^{x \times x}, \quad \mathbf{B}_{12} \in \mathbb{R}^{x \times(n-x)}, \quad \mathbf{B}_{21} \in \mathbb{R}^{(n-x) \times x}, \quad \mathbf{B}_{22} \in \mathbb{R}^{(n-x) \times(n-x)}$ are submatrices of $\mathbf{B}_{\text {red }} ; \mathbf{0}$ is a proper zero matrix; and $\mathbf{S}_{B x}=\operatorname{diag}\left\{S_{B 1}, \ldots, S_{B x}\right\}$.

Proof. Define right eigenvector of an arbitrary eigenvalue $\lambda$ for matrix $\mathbf{Z}_{\text {red }} \mathbf{S}_{B}$ as $\mathbf{v}=\left[\begin{array}{l}\mathbf{v}_{1} \\ \mathbf{v}_{2}\end{array}\right],\left(\mathbf{v}_{1} \in \mathbb{R}^{x}, \mathbf{v}_{2} \in \mathbb{R}^{(n-x)}\right)$, i.e.,

$$
\mathbf{Z}_{\text {red }}\left[\begin{array}{ll}
\mathbf{S}_{B x} & \\
& \mathbf{0}
\end{array}\right] \mathbf{v}=\lambda \mathbf{v}
$$


Since $\mathbf{Z}_{\text {red }}$ is a positive definite matrix, left-multiplying each side of (34) by $\mathbf{Z}_{\text {red }}^{-1}$ obtains

$$
\left[\begin{array}{ll}
\mathbf{S}_{B x} & \\
& \mathbf{0}
\end{array}\right] \mathbf{v}=\lambda \mathbf{B}_{\text {red }} \mathbf{v}
$$

Equation (35) shows that the eigenvalues for $\mathbf{Z}_{r e d} \mathbf{S}_{B}$ are equivalent to the eigenvalues for matrix pencil $\lambda \mathbf{B}_{\text {red }}-\mathbf{S}_{B}{ }^{[27]}$. Then, submitting $\mathbf{v}=\left[\begin{array}{l}\mathbf{v}_{1} \\ \mathbf{v}_{2}\end{array}\right]$ into (35) obtains that

$$
\left\{\begin{array}{l}
\lambda^{-1} \mathbf{v}_{1}=\mathbf{S}_{B x}^{-1} \mathbf{B}_{\text {redd }} \mathbf{v}_{1} \\
\lambda\left(\mathbf{B}_{21} \mathbf{v}_{1}+\mathbf{B}_{22} \mathbf{v}_{2}\right)=\mathbf{0}
\end{array}\right.
$$

Equation (36) shows that matrix pencil $\lambda \mathbf{B}_{r e d}-\mathbf{S}_{B}$ (or matrix $\mathbf{Z}_{r e d} \mathbf{S}_{B}$ ) has $(n-x)$ zero eigenvalues and $x$ non-zero eigenvalues that are the reciprocal of the eigenvalues of $\mathbf{S}_{B x}^{-1} \mathbf{B}_{\text {redx }}$. Since the eigenvalues of $\mathbf{S}_{B x}^{-1} \mathbf{B}_{\text {redx }}$ are positive, the proof is concluded $\mathbf{m}$.

Lemma 2 shows that $\lambda_{1}=\lambda_{\max }^{-1}\left(\mathbf{Z}_{r e d} \mathbf{S}_{B}\right)=\lambda_{\max }^{-1}\left(\mathbf{B}_{r e d}^{-1} \mathbf{S}_{B}\right)$, no matter that $S_{\mathrm{B} i}=0$ or $S_{\mathrm{B} i}>0(i \in\{1, \ldots, n\})$. With Lemma 2 , the optimization problem (29) can be rewritten as

$$
\begin{aligned}
& \max \sum_{i=1}^{n} S_{B i} \\
\text { s.t. } & \left\{\begin{array}{l}
\lambda_{\max }\left(\mathbf{Z}_{r e d} \mathbf{S}_{B}\right) \leq r \\
\mathbf{S}_{B} \succeq 0
\end{array}\right.
\end{aligned}
$$

where $r=1 / \lambda_{\min }^{\mathrm{U}} ;$ “ $\succeq$ ” is the Löwner partial order ${ }^{[24]}$; $\mathbf{S}_{B}=\operatorname{diag}\left(S_{B i}\right),(i=1, \ldots, n)$; and $\mathbf{S}_{B} \succeq 0$ denotes that $\mathbf{S}_{B}$ is a positive semi-definite matrix.

\section{B. Proposed SDP-based Method for GAC-SSSC Assessment}

Since the optimization problem (37) is nonlinear, it is challenging to efficiently solve (37) and find its optimal solution. To address this challenge, we convert (37) into an SDP and then prove that the SDP satisfies the pre-condition of Corollary 2.2.6 in [28], which shows the feasibility of finding the optimal solution.

To transform the optimization problem in (37) into an SDP, let $\mathbf{Z}_{\text {red }}^{1 / 2}=\left[\mathbf{a}_{1}, \ldots, \mathbf{a}_{n}\right]$, wherein $\mathbf{a}_{i} \in \mathbb{R}^{n},(i=1, \ldots, n)$. Since $\mathbf{Z}_{\text {red }}$ in (37) is positive-definite, $\mathbf{Z}_{\text {red }}^{1 / 2}$ is positive-definite. Then, we have $\mathbf{Z}_{\text {red }}^{-1 / 2} \mathbf{Z}_{\text {red }} \mathbf{S}_{B} \mathbf{Z}_{\text {red }}^{1 / 2}=\mathbf{Z}_{\text {red }}^{1 / 2} \mathbf{S}_{B} \mathbf{Z}_{\text {red }}^{1 / 2}$, and $\mathbf{Z}_{\text {red }} \mathbf{S}_{B}$ is similar to $\mathbf{Z}_{r e d}^{1 / 2} \mathbf{S}_{B} \mathbf{Z}_{\text {red }}^{1 / 2}$. This means that $\mathbf{Z}_{\text {red }} \mathbf{S}_{B}$ and $\mathbf{Z}_{\text {red }}^{1 / 2} \mathbf{S}_{B} \mathbf{Z}_{\text {red }}^{1 / 2}$ share the same eigenvalues. Thus, the optimization problem in (37) can be converted into a dual SDP in (38), which indicates that the optimization problem in (37) is convex.

$$
\begin{aligned}
& \max \sum_{i=1}^{n} S_{B i}
\end{aligned}
$$

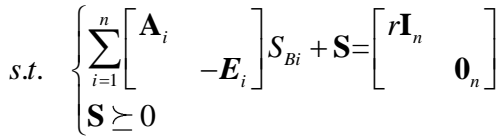

where $\mathbf{A}_{i}=\mathbf{a}_{i} \mathbf{a}_{i}^{T} ; \mathbf{I}_{n}$ and $\mathbf{0}_{n}$ are respectively a proper identity matrix and a proper zero matrix; and $\mathbf{S}$ is a slack matrix; $\boldsymbol{E}_{i}$ is a $n \times n$ square matrix, in which the $i$ th diagonal element is 1 and the other elements are zero. Note that $\mathbf{Z}_{r e d}^{1 / 2} \mathbf{S}_{B} \mathbf{Z}_{\text {red }}^{1 / 2}=\sum_{i=1}^{n} \mathbf{A}_{i} S_{B i}$.
The following proposition 1 can be proved to show that the dual SDP in (38) satisfies the pre-condition of the Corollary 2.2.6 in [28].

Proposition 1: Let $(\hat{\mathbf{y}}, \hat{\mathbf{S}}),\left(\hat{\mathbf{y}}=\left[\mathrm{S}_{B 1}, \ldots, \mathrm{S}_{B n}\right]^{\mathrm{T}}, \hat{\mathbf{S}} \succeq \mathbf{0}\right)$ be the feasible solution of the dual SDP in (38). And let $d^{*}$ be the upper limit of $\sum_{i=1}^{n} S_{B i}$ of the dual SDP in (38). Then, the dual SDP in (38) satisfies that:

a) The dual SDP has strictly feasible solutions. That is, the solution $(\hat{\mathbf{y}}, \hat{\mathbf{S}})$ to the dual SDP in (38) satisfies $\hat{\mathbf{S}} \succ \mathbf{0}$, wherein " $\succ$ " is the löwner partial order, and $\hat{\mathbf{S}}_{\succ} \mathbf{0}$ denotes that the slack matrix $\hat{\mathbf{S}}$ is a positive definite matrix;

b) The upper limit $d^{*}$ is finite.

Proof. Firstly, we prove that the dual SDP (38) has the strictly feasible solution, i.e., the proposition 1 a). To this end, $\hat{\mathbf{y}}$ is set as $\varepsilon \mathbf{1}$, wherein the arbitrary element $\varepsilon$ satisfies that $\varepsilon \geq 0$, and the elements of $\mathbf{1} \in \mathbb{R}^{n}$ are all one. Define the eigenvalues of $\mathbf{Z}_{r e d}$ as $\lambda_{z i},(i=1, \ldots, n)$. Then, the eigenvalues of $\mathbf{Z}_{r e d}^{1 / 2} \mathbf{S}_{B} \mathbf{Z}_{\text {red }}^{1 / 2}$ are given as $\varepsilon \lambda_{z i},(i=1, \ldots, n)$. Since $\mathbf{Z}_{r e d}$ is a positive definite matrix, we have $\lambda_{z i}>0,(i=1, \ldots, n)$. We define the maximal eigenvalue of $\mathbf{Z}_{\text {red }}$ as $\lambda_{z \max }$. Then, the maximal eigenvalue of $\mathbf{Z}_{r e d}^{1 / 2} \mathbf{S}_{B} \mathbf{Z}_{r e d}^{1 / 2}$ is $\varepsilon \lambda_{z \max }$. According to (38), when $\hat{\mathbf{y}}=\varepsilon \mathbf{1}$, the slack matrix $\hat{\mathbf{S}}$ can be rewritten as

$$
\hat{\mathbf{S}}=\left[\begin{array}{ll}
r \mathbf{I}_{n}-\varepsilon \mathbf{Z}_{\text {red }} & \\
& \varepsilon \mathbf{I}_{n}
\end{array}\right]
$$

To ensure $\hat{\mathbf{S}} \succ \mathbf{0}$ in (39), the following inequation should be satisfied

$$
\left\{\begin{array}{l}
r>\varepsilon \lambda_{\text {max }} \\
\varepsilon>0
\end{array} \Rightarrow 0<\varepsilon<r / \lambda_{\text {max }}\right.
$$

Thus, there is always a strictly feasible solution $\left(\varepsilon \mathbf{1}, r \mathbf{I}_{n}-\varepsilon \mathbf{Z}_{r e d}\right),\left(0<\varepsilon<r / \lambda_{z \max }\right)$ for the dual SDP in (38).

Secondly, we prove the proposition $1 \mathrm{~b}$ ). According to (38), the slack matrix $\mathbf{S}$ satisfies that

$$
\mathbf{S}=\left[\begin{array}{ll}
r \mathbf{I}_{n} & \\
& \mathbf{0}_{n}
\end{array}\right]-\sum_{i=1}^{n}\left[\begin{array}{ll}
\mathbf{A}_{i} & \\
& -\boldsymbol{E}_{i}
\end{array}\right] S_{B i}=\left[\begin{array}{ll}
r \mathbf{I}_{n}-\mathbf{Z}_{r e d}^{1 / 2} \mathbf{S}_{B} \mathbf{Z}_{r e d}^{1 / 2} & \\
& \mathbf{S}_{B}
\end{array}\right] \succeq 0
$$

Equation (41) shows that $\mathbf{S}_{B}$ is a positive semi-definite matrix and the spectral norm of the positive semi-definite matrix $\mathbf{Z}_{r e d}^{1 / 2} \mathbf{S}_{B} \mathbf{Z}_{r e d}^{1 / 2}\left(\right.$ or $\mathbf{Z}_{\text {red }} \mathbf{S}_{B}$ ) satisfies that

$$
\left\|\mathbf{Z}_{r e d}^{1 / 2} \mathbf{S}_{B} \mathbf{Z}_{\text {red }}^{1 / 2}\right\|_{2}=\left\|\mathbf{Z}_{\text {red }} \mathbf{S}_{B}\right\|_{2} \leq r
$$

where \|\|$_{2}$ is the spectral norm of an arbitrary matrix.

Equation (42) can be furtherly converted as that

$$
\left\|\mathbf{Z}_{\text {red }} \mathbf{S}_{B}\right\|_{2} \leq\left\|\mathbf{Z}_{\text {red }}\right\|_{2}\left\|\mathbf{S}_{B}\right\|_{2}=\lambda_{z \max } S_{B \text { max }} \leq r \Rightarrow S_{B \max } \leq r / \lambda_{z \max }
$$

where $S_{B \max }$ is the maximal value of $S_{B i}(i=1, \ldots, n)$ and $\lambda_{\text {zmax }}$ is the maximal eigenvalue of $\mathbf{Z}_{\text {red }}$.

Based on (43), the sum of $S_{B i}(i=1, \ldots, n)$ satisfies that

$$
\sum_{i=1}^{n} S_{B i} \leq n S_{B \max } \leq n r / \lambda_{z \max }
$$

Therefore, the upper limit $d^{*}$ of the dual SDP in (38) is finite. Thus, the proof is concluded $\mathbf{m}$. 
Since Proposition 1 is true, according to Corollary 2.2.6 in [28], we can conclude that the optimal solution to the dual SDP in (38) can be found.

Remark 2. The optimal solution to the dual SDP in (38) (or the optimization problem in (37)) can be found.

Since the software solver for the SDP is very mature, such as SeDuMi ${ }^{[29]}$ and YALMIP ${ }^{[30]}$, the details of the algorithm for solving the dual SDP in (38) is not presented here. In the next section, the YALMIP will be used as the solver for the dual SDP in (38).

\section{Simulation VALIDATION}

In this section, the effectiveness of the proposed SDP-based method for GAC-SSSC assessment is verified on a modified 39-node system as shown in Fig. 4. In this system, nodes 1 11 are nodes for potential CBR integration, and node 39 is infinite node. The network topology of the system is the same as that of the standard IEEE 39-node system, and its line parameters can be found in [31]. The parameters of integrated CBRs are presented in Appendix. Due to page limitation, we will focus on the heterogeneous MCS in this section, since the homogeneous MCS is a special case of the heterogeneous MCS for the GAC-SSSC assessment using the proposed SDP-based method. To form such a heterogeneous MCS, 9 CBRs are placed at nodes 1 9 in the modified IEEE 39-node system, and the following settings are used for these CBRs: the active power controls of CBRs at nodes $1 \sim 4$ are set as constant active power control, and those of the other CBRs are set as dc-voltage control; the PLL proportional-integrational (PI) control parameters of CBRs at nodes $1 \sim 4$ are set as " 26,7800 ", and those of the CBR at node 9 as "35 7000".

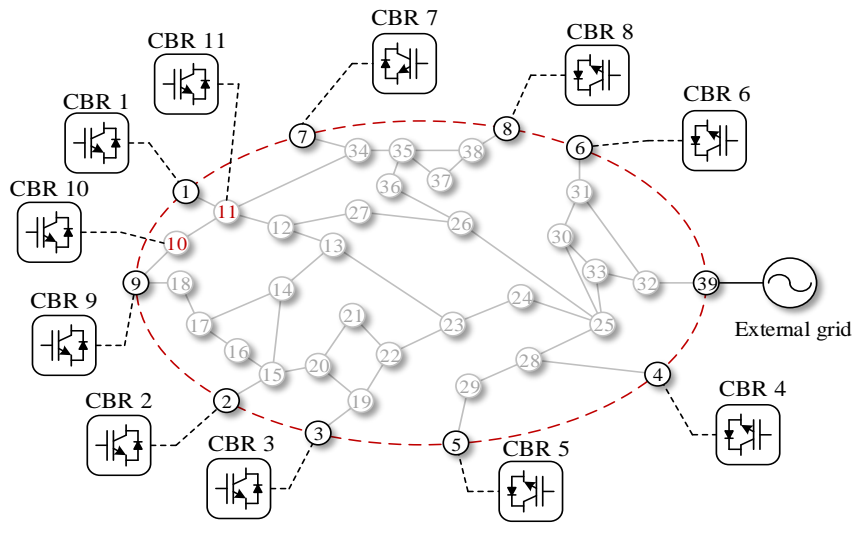

Fig. 4 One-line diagram of a modifying 39-node system.

\section{A. Small-signal Stability Analysis of MCSs Based on Smallest Network Eigenvalue}

The proposed SDP-based method for GAC-SSSC assessment is based on the smallest network eigenvalue $\lambda_{1}$, which is derived in Section III to evaluate the small-signal stability of heterogeneous MCSs. Before validating the proposed SDP-based method for GAC-SSSC assessment, we first verify the efficacy of evaluating small-signal stability based on the smallest network eigenvalue $\lambda_{1}$ by eigenvalue analysis on the modified IEEE 39-node system.

In the eigenvalue analysis, several cases are created in the system by increasing the rated capacity of CBR1 (i.e., $S_{B 1}$ ) from 0.5 p.u. to 5.0 p.u. while keeping the capacities of the other CBRs as 1.0 p.u.. Accordingly, we evaluate the changes of the smallest network eigenvalue $\lambda_{1}$, and the resulting loci of the dominant eigenvalues of the system, and the damping ratios of the system, which are shown in Fig. 5(a) and Fig. 5(b).

It can be observed from Fig. 5 that the smallest network eigenvalues $\lambda_{1}$ can effectively quantify the small-signal stability of the system. As shown in Fig. 5 (a), when $\lambda_{1}=2.35$, the loci of the dominant eigenvalues of the system are exactly at the imaginary axis, which indicates the system is critically stable. When $\lambda_{1}>2.35$, the loci of the dominant eigenvalues of the system are in the left-half of the complex plane, which suggests the system is stable. When $\lambda_{1}<2.35$, the loci of the dominant eigenvalues of the system are in the right-half of the complex plane, which means the system is unstable. The similar observation can also be obtained from Fig. 5(b) in terms of the damping ratio. In this figure, the red curve is the change in the damping ratio of the modified IEEE 39-node system when increasing $S_{\mathrm{B} 1}$ from 0.5 p.u. to 5.0 p.u and keeping the capacities of the other CBRs as 1.0 p.u. As shown in Fig. 5(b), when $\lambda_{1}=2.35$, the damping ratio of the system is zero, which means that the system is critically stable; when $\lambda_{1}>2.35$ (or $\lambda_{1}<2.35$ ), the loci of the damping ratio of the system is larger (or smaller) than zero, which indicates the system is stable (or unstable). Thus, the results also validate the effectiveness of using the smallest network eigenvalue $\lambda_{1}$ to assess the small-signal stability of the system.

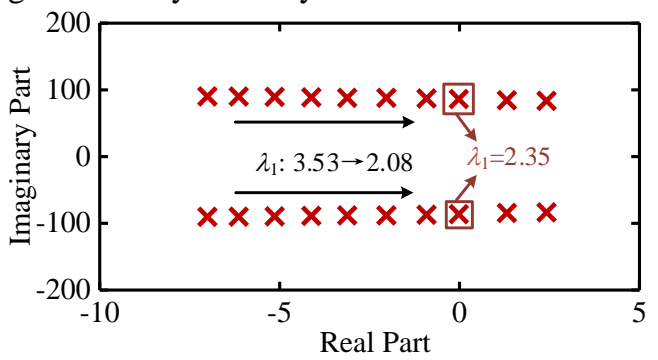

(a)

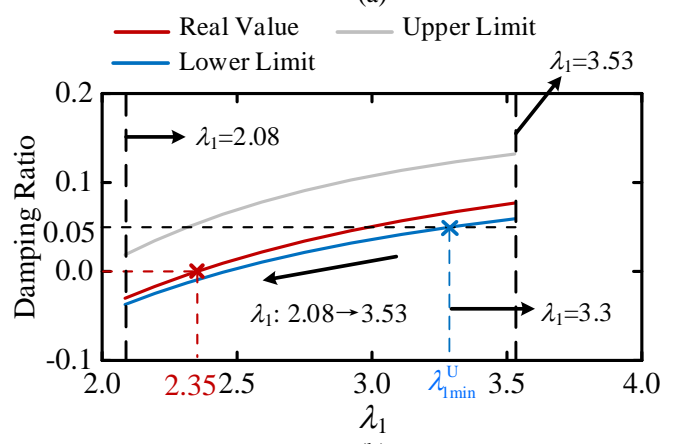

(b)

Fig. 5 The changes of the loci of dominant eigenvalues and corresponding damping ratio for the modified IEEE 39-node system when reducing the smallest network eigenvalue $\lambda_{1}$ : (a) the change of loci of dominant eigenvalues with varying $\lambda_{1}$; (b) the change of the loci of damping ratio(red line "—”), and its upper (grey line “_”) and lower limits (blue line “—”) with varying $\lambda_{1}$. 
Moreover, it can be observed from Fig. 5(b) that the small-signal stability of the modified IEEE 39-node system is bounded by its upper and lower limits. According to the analysis in Section III.B, it is known that the modified IEEE 39-node system can be converted into an equivalent homogeneous system for the small-signal stability analysis. The stability of the equivalent homogeneous system is bounded by the dynamics of equivalent $\mathrm{SCS}_{1,1}$ and $\mathrm{SCS}_{1, \mathrm{n}}$, which are corresponding to the most unstable and stable SCSs. Thus, the stability of the modified IEEE 39-node system is also bounded by the dynamics of $\mathrm{SCS}_{1,1}$ and $\mathrm{SCS}_{1,9}$. In Fig. 5(b), the blue and grey curves are the damping ratios, which are determined by the $\mathrm{SCS}_{1,1}$ and $\mathrm{SCS}_{1,9}$. We can see from Fig. 5(b) that the red curve is between the gray and blue curves, which indicates the stability of the modified IEEE 39-node system is bounded. These results are consistent with the theoretical analysis in Section III.B and also verify the effectiveness of using $\lambda_{1}$ to quantify the small-signal stability of MCSs.

\section{B. Proposed SDP-based Method for GAC-SSSC Assessment in MCSs}

To verify the effectiveness of the proposed SDP-based method for the GAC-SSSC assessment in MCSs while demonstrating the capability of the proposed method to consider the passive nodes in the GAC-SSSC assessment, the modified IEEE 39-node system used in Section V.A is used here, but two additional passive nodes 10 and 11 in the system are included as the potential points for CBR integration. The control parameters of additional CBRs at nodes 10 and 11 are the same as those of the CBR at node 9 in the system. Besides, when the small-signal stability limit in terms of damping ratio $\zeta_{\text {min }}$ is set as 0.05 and $\lambda^{\mathrm{U}}{ }_{1 \text { min }}$ is set as 3.3 p.u., which can be observed from Fig. 5(b). In the proposed method, the established SDP in (38) is solved by the solver YALMIP to determine the GAC-SSSC of all CBRs in the modified IEEE 39-node system.

To demonstrate its solution superiority, the proposed method is compared with an equal-capacity-allocation method (ECAM) for GAC-SSSC assessment. In the ECAM, the capacities of all CBRs are increased equally until the system stability reaches the small-signal stability limit $\zeta_{\min }$. Based on the ECAM, the determined GAC-SSSC is 10.285 p.u., and each CBR has the same capacity equal to 0.935 p.u., which are presented in Table 1. By using the proposed SDP-based method, the determined GAC-SSSC is 11.813 p.u., and the resulting optimal capacity allocation of CBRs is given in Table 2. Table 2 shows that the capacities of CBR1 and CBR10 are zero. This indicates that it is necessary to consider the scenario, where the capacities of partial CBRs are zero when assessing the GAC-SSSC of CBRs, as discussed in Section IV.A.

Table 1 Capacity allocation for all CBRs determined by the ECAM

\begin{tabular}{cccccc}
\hline CBR1 & CBR2 & CBR3 & CBR4 & CBR5 & CBR6 \\
0.935 & 0.935 & 0.935 & 0.935 & 0.935 & 0.935 \\
\hline CBR7 & CBR8 & CBR9 & CBR10 & CBR11 & \\
0.935 & 0.935 & 0.935 & 0.935 & 0.935 & \\
\hline
\end{tabular}

Table 2 Optimal capacity allocation for all CBRs determined by the proposed SDP-based method

\begin{tabular}{cccccc}
\hline CBR1 & CBR2 & CBR3 & CBR4 & CBR5 & CBR6 \\
0 & 0.392 & 0.563 & 1.301 & 0.581 & 7.025 \\
\hline CBR7 & CBR8 & CBR9 & CBR10 & CBR11 & \\
0.11 & 0.33 & 0.151 & 0 & 1.36 & \\
\hline
\end{tabular}

Under the capacity allocation of CBRs shown in Tables 1 and 2 , the system stability is checked by time-domain simulation. In the simulation, a disturbance is applied to the infinite-bus at $t=1 \mathrm{~s}$ to cause 0.05 p.u. of voltage drops and then the voltage is recovered at $t=1.05 \mathrm{~s}$. The time-domain responses of active power outputs of all CBRs in the system are shown in Fig. 6. To demonstrate the importance of the small-signal stability constraints in the GAC assessment, Fig. 7 also shows the time-domain responses when active power outputs of all CBRs are set as 1.2 p.u, which are not the solutions of the proposed SDP-based method and the ECAM.
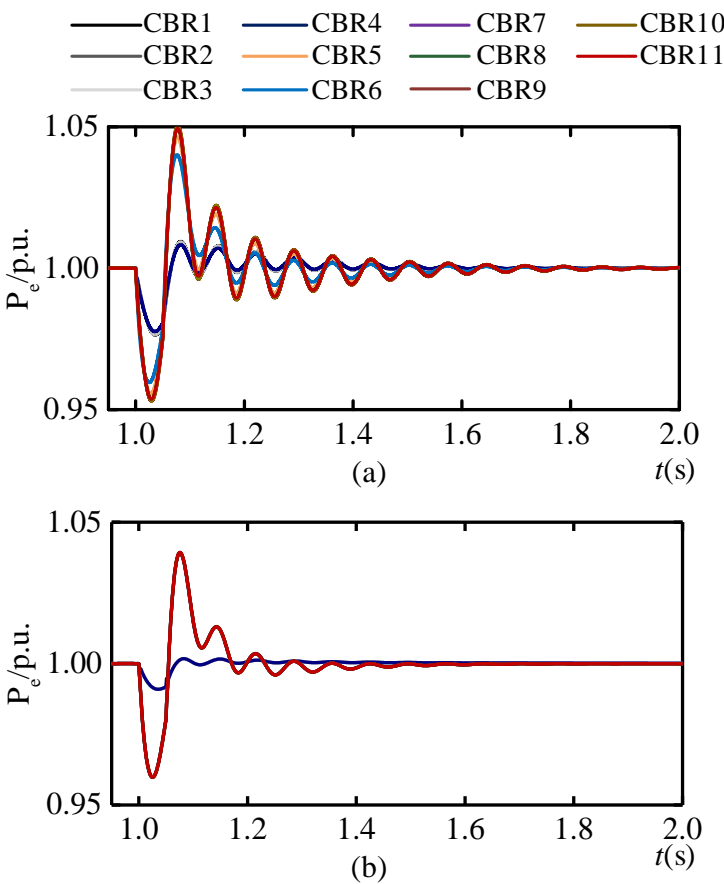

Fig. 6 Time-domain responses of active power of all CBRs with capacity allocations determined by (a) the ECAM and (b) the established SDP in the modified IEEE 39-node system.

It can be seen from Fig. 6 and Fig. 7 that the proposed SDP-based method and the ECAM can effectively determine the grid-accommodable capacity of renewable generation while maintaining the small-signal stability, but the proposed SDP-based method is superior to the ECAM. By comparing Fig. 6 and Fig. 7, it can be seen that all CBRs are stable in the system under the CBR capacity allocation of either Tables 1 or 2, but these CBRs may be not stable when their capacity allocations are different from those presented in Tables 1 or 2. Further, it can be seen from Fig. 6 that the convergence speed of the time-domain responses in Fig. 6(b) is faster than that in Fig. 6(a), which indicates the system is more stable under the CBR capacity allocation of Table 2 determined by the proposed SDP-based method than that of Table 1 determined by the ECAM. This is because the 
small-signal stability limit used in the established SDP is the upper limit for heterogeneous MCSs. More importantly, the GAC-SSSC (i.e., 11.813 p.u.) determined by the proposed SDP-based method is larger than that (i.e., 10.285 p.u.) decided by the ECAM. Thus, these results validate the efficacy of the proposed SDP-based method.

Also, the loci of the gap between the feasible solutions of the dual SDP (38) and its corresponding primal SDP in the proposed method is evaluated to verify the established SDP can find the optimal solution in MCSs. Fig. 8 shows the loci of the gap in semi-log plot on a base-10 logarithmic scale when increasing iterations. It can be observed from this figure that the gap is reducing with the increased iterations. Particularly, the gap is smaller than $10^{-6}$ at the 7th iteration, which is approximately equal to zero. According to [28], it is known that the dual SDP and its corresponding primal SDP find their optimal solutions when the gap of their solutions is zero. Thus, the used solver finds the optimal solution of the dual SDP (38), which is consistent with the theoretical analysis in Section IV.B.

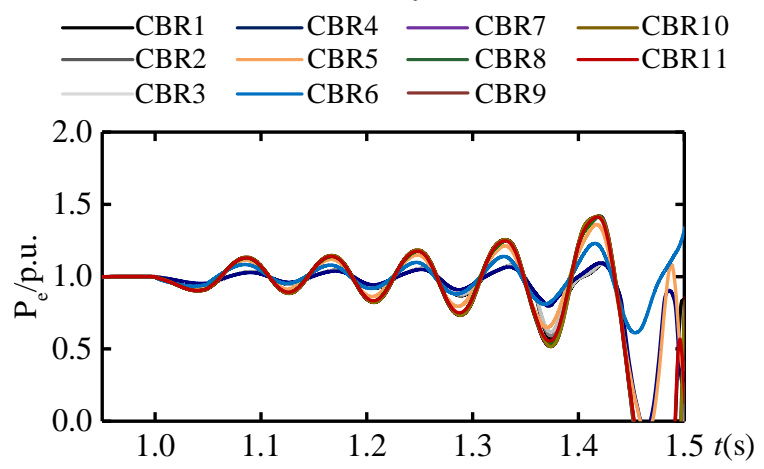

Fig. 7 Time-domain responses of active power of all CBRs when the capacities of all CBRs are set as 1.2 p.u. in the modified IEEE 39-node system.

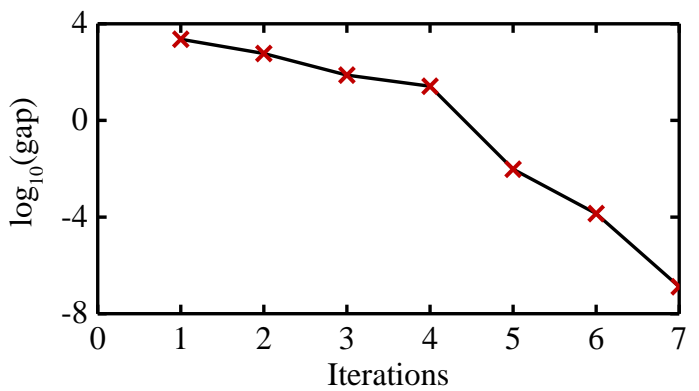

Fig. 8 Loci of the gap between the feasible solutions of the dual SDP (38) and its corresponding primal SDP when increasing iterations.

\section{CONCLUSIONS}

In this paper, an SDP-based method was proposed for evaluating the GAC-SSSC of CBRs in MCSs. To reduce the complexity for assessing GAC-SSSC in a MCS with large-scale CBRs, the small-signal stability constraints were formulated in the established SDP based on the smallest network eigenvalue (i.e., the smallest eigenvalue of a weighted Laplacian matrix of the power network in a MCS). It was derived that the smallest network eigenvalue can quantify the small-signal stability of a MCS. By focusing on the smallest network eigenvalue, we circumvent the step of deriving the complex system model for the small-signal stability assessment in MCSs. Moreover, it was proved that the derived SDP can find the optimal solution. The effectiveness of the proposed SDP-based method was demonstrated on a modified 39-node power system. The proposed method is helpful for grid planners to improve the utilization efficiency of renewable generation by enhancing the grid-accommodable capacity of renewable generation while maintaining the small-signal stability in MCSs with large-scale integration of CBRs.

\section{APPENDIX}

Table. I Control parameters of an CBR (per-unit values)

Filter inductance $L_{\mathrm{f}}$ and capacitance $C_{\mathrm{f}}$, dc capacitance $C_{\mathrm{dc}}: 0.05,0.05,0.038$

Transfer function of the current control loop $H_{\mathrm{i}}(s): 1+10 / s$

Transfer function of the dc voltage control loop $H_{\mathrm{dc}}(s): 1+5 / s$

Transfer function of the constant active power control loop $H_{\mathrm{p}}(s): 2+15 / s$

Transfer function of the voltage feedforward filter $G_{\mathrm{FF}}(s): 1 /(1+0.01 s)$

Transfer function of the PLL $H_{\mathrm{pll}}(s):(30+7500 / s) / s$

Parameters of active power output and $q$-axis current reference: 1,0

\section{REFERENCES}

[1] F. Milano, F. Dörfler, G. Hug, D. J. Hill, and G. Verbič, "Foundations and challenges of low-inertia systems," in 2018 Power Systems Computation Conference (PSCC). IEEE, pp. 1-25, 2018.

[2] Q. Peng, Q. Jiang, H. Yang, T. Liu, H. Wang, and F. Blaabjerg, "On the stability of power electronics-dominated systems: Challenges and potential solutions," IEEE Trans. Ind. Appl., vol. 55, no. 6, pp. 76577670, Nov. 2019.

[3] Y. Li, L. Fan, and Z. Miao, "Wind in weak grids: Low-frequency oscillations, subsynchronous oscillations, and torsional interactions," IEEE Trans. Power syst., vol. 35, no. 1, pp. 109-118, Jan. 2020.

[4] M. Zhao, X. Yuan, J. Hu, and Y. Yan, "Voltage dynamics of current control time-scale in a VSC-connected weak grid," IEEE Trans. Power syst., vol. 31, no. 4, pp. 2925-2937, Jul. 2016.

[5] L. Guan, J. Yao, R. Liu, and et al., "Small-signal stability analysis and enhanced control strategy for VSC system during weak-grid asymmetric Faults," IEEE Trans. Sustain. Energy, vol. 12, no. 4, pp. 2074-2085, Oct. 2021.

[6] H. Tan, Z. Ren, W. Yan, and et al., "A wind power accommodation capability assessment method for multi-energy microgrids," IEEE Trans. Sustain. Energy, vol. 12, no. 4, pp. 2482-2492, Oct. 2021.

[7] Y. Huo, F. Bouffard, G. Joos, "Spatio-temporal flexibility management in low-carbon power systems," IEEE Trans. Sustain. Energy, vol. 11, no. 4, pp. 2593-2605, Oct. 2020.

[8] C. E. Murillo-Sánchez, R. D. Zimmerman, C. L. Anderson, et al., "Secure planning and operations of systems with stochastic sources, energy storage, and active demand," IEEE Trans. Smart Grid, vol. 4, no. 4, pp. 2220-2229, Dec. 2013.

[9] Y. Ding, P. Wang, L. Goel, et al. "Long-term reserve expansion of power systems with high wind power penetration using universal generating function methods," IEEE Trans. Power syst., vol. 26, no. 2, pp. 766-774, May. 2011.

[10] Q. Xu, C. Kang, N. Zhang, et al., "A probabilistic method for determining grid-accommodable wind power capacity based on multiscenario system operation simulation," IEEE Trans. Smart Grid, vol. 7, no. 1, pp. 400-409, Jan. 2016.

[11] E. Sáiz-Marín, E. Lobato, I. Egido, "Local hosting capacity increase by means of wind farm voltage control provision," IEEE Trans. Power syst., vol. 29, no. 4, pp. 1731-1738, Jul. 2014.

[12] H. Ayres, W. Freitas, M. Almeida, "Method for determining the maximum allowable penetration level of distributed generation without steady-state voltage violations," IET Gener. Trans. Distrib., vol. 4, no. 4, pp. 495-508, 2010.

[13] X. Dong, C. Kang, Y. Ding, C. Wang, "Estimating the wind power integration threshold considering electro-thermal coupling of overhead transmission lines," IEEE Trans. Power syst., vol. 34, no. 5, pp. 3349-3358, Sep. 2019. 
[14] J. Liu, J. Wen, J. Fang, and et al. "Impact of power grid strength and PLL parameters on stability of grid-connected DFIG wind farm," IEEE Trans. Sustain. Energy, vol. 11, no. 1, Jan. 2020.

[15] L. Fan, "Modeling type-4 wind in weak grids," IEEE Trans. Sustain. Energy, vol. 10, no. 2, Apr. 2019.

[16] J. Zhou, H. Ding, S. Fan, Y. Zhang, and A. Gole, "Impact of short-circuit ratio and phase-locked-loop parameters on the small-signal behavior of a VSC-HVDC converter," IEEE Trans. Power Del., vol. 29, no. 5, pp. 2287-2296, Oct. 2014.

[17] W. Du, Y. Wang, H. Wang, and et al., "Small-disturbance stability limit of a grid-connected wind farm with PMSGs in the timescale of DC voltage dynamics," IEEE Trans. Power syst., vol. 36, no. 3, pp. 2366-2379, May. 2021.

[18] W. Du, W. Dong, and H. F. Wang, "Small-signal stability limit of a grid-connected PMSG wind farm dominated by the dynamics of PLLs," IEEE Trans. Power syst., vol. 35, no. 3, pp. 2093-2107, May. 2020.

[19] H. Liu, X. Xie, J. He, T. Xu, Z. Yu, C. Wang, and C. Zhang, "Subsynchronous interaction between direct-drive PMSG based wind farms and weak AC networks," IEEE Trans. Power syst., vol. 32, no. 6, pp. 4708-4720, Nov. 2017.

[20] Y. Lin, J. Eto, B. Johnson, and et al., "Research Roadmap on Grid-Forming Inverters," United States, 2020, https://www.osti.gov/ servlets/purl/1721727.

[21] P. Kundur, N. J. Balu, and M. G. Lauby, Power system stability and control. McGraw-hill, New York, 1994.

[22] W. Dong, H. Xin, D. Wu, and L. Huang, "Small signal stability analysis of multi-infeed power electronic systems based on grid strength assessment," IEEE Trans. Power syst., vol. 34, no. 2, pp. 1393-1403, May. 2019.

[23] W. Du, Y. Wang, H. Wang, X. Xiao, "Reduced-order method for detecting the risk and tracing the sources of small-signal oscillatory instability in a power system with a large number of wind farms," IEEE Trans. Power Electron., vol. 36, no. 2, pp. 1563-1582, Mar. 2021.

[24] R. A. Horn and C. R. Johnson, Matrix analysis. Cambridge university press, 2012.

[25] B. Wen, D. Boroyevich, R. Burgos, P. Mattavelli, and Z. Shen, "Analysis of D-Q small-signal impedance of grid-tied inverters," IEEE Trans. Power Electron., vol. 31, no. 1, pp. 675-687, Jan. 2016.

[26] G. W. Stewart, Matrix perturbation theory. Citeseer, 1990.

[27] G. Tzounas, I. Dassios, and F. Milano, "Modal participation factors of algebraic variables," IEEE Trans. Power syst., vol. 35, no. 1, pp. 742750, Jan. 2020.

[28] C. Helmberg, Semidefinite Programming for Combinatorial Optimization. Konrad-Zuse-Zentrum für Informationstechnik Berlin, 2000.

[29] J. Sturm, "Using SeDuMi 1.02, a MATLAB toolbox for optimization over symmetric cones," Optimization Methods and Software, vol. 11, pp. 625-653, 1999.

[30] J. Löfberg, "YALMIP: a toolbox for modeling and optimization in MATLAB," in IEEE International Symposium on Computer Aided Control Systems Design (CACSD). IEEE, pp. 284-289, 2005.

[31] L. Huang, H. Xin, W. Dong, and F. Dörfler, "Impacts of grid structure on PLL-synchronization stability of converter-integrated power systems," arXiv preprint arXiv:1903.05489, 2019. 